OPEN ACCESS

Edited by:

Qiliang Cai,

Fudan University, China

Reviewed by:

John Sinclair,

University of Cambridge,

United Kingdom

Juliet Spencer,

Texas Woman's University,

United States

*Correspondence:

Jennifer N. Styles

jnstyles@live.unc.edu

Specialty section:

This article was submitted to

Virus and Host

a section of the journa

Frontiers in Cellular and Infection

Microbiology

Received: 03 March 2020

Accepted: 02 June 2020

Published: 09 July 2020

Citation:

Styles JN, Converse RR, Griffin SM,

Wade TJ, Klein E,

Nylander-French LA, Stewart JR,

Sams E, Hudgens E and Egorov Al

(2020) Human Cytomegalovirus

Infections Are Associated With

Elevated Biomarkers of Vascular Injury.

Front. Cell. Infect. Microbiol. 10:334.

doi: 10.3389/fcimb.2020.00334

\section{Human Cytomegalovirus Infections Are Associated With Elevated Biomarkers of Vascular Injury}

\author{
Jennifer N. Styles ${ }^{1,2 *}$, Reagan R. Converse ${ }^{1}$, Shannon M. Griffin ${ }^{3}$, Timothy J. Wade ${ }^{1}$, \\ Elizabeth Klein ${ }^{4}$, Leena A. Nylander-French ${ }^{2}$, Jill R. Stewart ${ }^{2}$, Elizabeth Sams ${ }^{1}$, \\ Edward Hudgens ${ }^{1}$ and Andrey I. Egorov ${ }^{1}$ \\ 1 United States Environmental Protection Agency, Office of Research and Development, Research Triangle Park, NC, \\ United States, ${ }^{2}$ Department of Environmental Sciences and Engineering, Gillings School of Global Public Health, The \\ University of North Carolina, Chapel Hill, NC, United States, ${ }^{3}$ United States Environmental Protection Agency, Office of \\ Research and Development, Cincinnati, OH, United States, ${ }^{4}$ ORAU Student Services Contractor to US EPA, Chapel Hill, NC, \\ United States
}

Background: Human cytomegalovirus (HCMV) infects $~ 50 \%$ of adults in the United States. HCMV infections may cause vascular inflammation leading to cardiovascular disease, but the existing evidence is inconsistent.

Objective: We investigated demographic predictors of HCMV infection and explored associations between HCMV infection status, the intensity of anti-HCMV Immunoglobulin $G$ (IgG) antibody response, and biomarkers of inflammation and endothelial function which are known predictors of cardiovascular disease.

Methods: We conducted a cross-sectional study of 694 adults residing in the Raleigh-Durham-Chapel Hill, NC metropolitan area. Serum samples were tested for IgG antibody response to HCMV, and for biomarkers of vascular injury including soluble intercellular adhesion molecule 1 (SICAM-1), soluble vascular cell adhesion molecule 1 (sVCAM-1), C-reactive protein (CRP), and serum amyloid A (SAA). Associations between HCMV and biomarker levels were analyzed using two approaches with HCMV serostatus modeled as a binary variable and as an ordinal variable with five categories comprised of seronegative individuals and quartiles of anti-HCMV antibody responses in seropositive individuals.

Results: HCMV seroprevalence in the study population was 56\%. Increased body mass index, increased age, female gender, racial/ethnic minority status, and current smoking were significantly associated with HCMV seropositivity in a multivariate regression analysis. HCMV seropositivity was also associated with 9\% (95\% confidence interval 4-15\%) and 20\% (0.3-44\%) increases in median levels of sICAM-1 and CRP, respectively, after adjusting for covariates. The association between HCMV seropositivity and median levels of SVCAM-1 and SAA were positive but not statistically significant. Significant positive associations were observed between the intensity of anti-HCMV IgG responses and levels of sICAM-1 and SVCAM-1 ( $p$-values 0.0008 and 0.04 for linear trend, respectively). To our knowledge, this is the first epidemiological study to show a 
relationship between anti-HCMV IgG responses and vascular injury biomarkers sICAM-1 and SVCAM-1 in the general population.

Conclusion: HCMV infections are associated with vascular injury and inflammation biomarkers in adult residents of North Carolina.

Keywords: cardiovascular disease, cytomegalovirus-HCMV, serum amyloid A, vascular injury, biomarkers, intercellular adhesion molecular-1 (ICAM-1), C-reactive protein, vascular cell adhesion molecule-1 (VCAM-1)

\section{INTRODUCTION}

Human cytomegalovirus (HCMV) is a member of the herpes virus family. The virus can be transmitted through person-toperson contacts involving exchange of infected bodily fluids such as blood, saliva, breast milk, urine, oropharyngeal secretions, cervical, vaginal secretions, and seminal fluid; sexual contact is a frequent mode of transmission (Cannon et al., 2010). HCMV causes a long-term latent infection with periodic flareups. In a new HCMV infection, immunoglobulin M (IgM) is produced for 3-6 months, after which HCMV-specific IgM can no longer be detected in serum (Prince and Lapé-Nixon, 2014). During this 3-6-month period, the immunoglobulin G (IgG) response is established and remains high throughout the life-long latency of an HCMV infection. Serum IgG tests are widely used to determine HCMV infection status. AntiHCMV IgG response increases during reactivation or reinfection of HCMV (Mehta et al., 2000; Carlson et al., 2010; Prince and Lapé-Nixon, 2014). The IgG seroprevalence of HCMV in the US was $50.4 \%$ between 1999 and 2004 according to the National Health and Nutrition Examination Survey (NHANES) (Bate et al., 2010).

Infection with HCMV is an important public health concern (Schleiss, 2008). It causes $\sim 30,000(0.7 \%)$ congenital infections annually in the US resulting in hearing loss, speech and developmental disabilities, or death for infected children (Ross and Boppana, 2004; Dollard et al., 2007; Martin et al., 2010). In adults with non-compromised immune systems, HCMV infection may occur without symptoms although some individuals may have transient mild mononucleosis-like symptoms (Lancini et al., 2014). Chronic HCMV infections have been associated with increased risks of cardiovascular disease (CVD), immune dysfunction, frailty, and depression (Simanek et al., 2014; Collins-Mcmillen et al., 2018). A proposed mechanism for the development of $\mathrm{CVD}$ as a result of HCMV infection is the increased conversion of prothrombin to thrombin, a clot forming agent, either directly or indirectly through increased inflammation (Popović et al., 2012). Previous in vivo and in vitro studies of CVD development due to HCMV infection have produced mixed results. High prevalence of HCMV viruses has been demonstrated in carotid atherosclerotic plaques (Yaiw et al., 2013). Some studies have also demonstrated positive associations between HCMV infection and various biomarkers of inflammation, endothelial function and vascular injury, such as C-reactive protein (CRP), soluble intercellular adhesion molecule 1 (sICAM-1), and soluble vascular cell adhesion molecule 1 (sVCAM-1). Several epidemiological studies have linked HCMV seropositivity with increased levels of CRP (Fateh-Moghadam et al., 2003; Betjes et al., 2007; Simanek et al., 2011); however, this association was not observed in two other studies, potentially due to the non-specific nature of inflammatory biomarker CRP (Simanek et al., 2014; Terrazzini et al., 2014). Three small (>100 participants) epidemiologic studies found associations between anti-HCMV IgG responses and increased levels of sICAM-1 and sVCAM-1 in serum samples in renal transplant patients and symptomatic individuals with primary HCMV infections, but not in asymptomatic healthy individuals (Nordoy et al., 2000; Eriksson et al., 2001; Lee et al., 2019). In-vitro experiments demonstrated that cellular adhesion molecules SVCAM-1 and sICAM-1 are released when endothelial cells are infected with HCMV (Popović et al., 2012). Meta-analysis of prospective studies demonstrated that HCMV infections were associated with an increased risk of CVD although some cohort studies included in this analysis detected no such association (Haider et al., 2002).

The objective of this study was to assess if HCMV infection affects four selected biomarkers of inflammation and endothelial function which are known predictors of cardiovascular morbidity and mortality: CRP, serum amyloid A (SAA), sVCAM-1, and sICAM-1. CRP and SAA are markers of acute inflammation. Chronically elevated CRP is linked with coronary artery disease however, elevated serum levels of CRP or SAA can also indicate other health conditions such as cancer (Zakynthinos and Pappa, 2009; Kaptoge et al., 2012; Baumann et al., 2017). VCAM-1 and ICAM-1 are biomarkers of endothelial function. These molecules are released into circulation by vascular endothelial cells in response to inflammation. Their useful functions include stable mediation of leukocyte adherence to the vascular endothelium and transmigration. However, elevated concentrations of VCAM-1 and ICAM-1 are linked with the development of atherosclerosis (Blankenberg et al., 2003; Galkina and Ley, 2007; Zakynthinos and Pappa, 2009).

\section{MATERIALS AND METHODS Study Population}

The ethical considerations of this study were reviewed and approved by the University of North Carolina Institutional Review Board (UNC IRB ref. \# 12-2600). All participants provided written consent prior to data collection.

This study was comprised of two subsets of a cross sectional study of 703 adults ( $>18$ years of age) in the Raleigh-DurhamChapel Hill metropolitan area in North Carolina in 2013. The 
first sub-set was a convenience sample study $\left(n_{1}=352\right)$ recruited by the United States Environmental Protection Agency (US EPA) through local advertising (hereon referred to as US EPA subset). The second subset of individuals $\left(n_{2}=351\right)$ was recruited for the Sample Collection Registry for Quality Control of Biological and Environmental Specimens and Assay Development and Testing study protocol number 10-E-0063, ClinicalTrials.gov identifier NCT01087307 at the National Institute of Environmental Health Sciences (NIEHS), Research Triangle Park, NC (hereon referred to as NIEHS subset).

Serum samples and questionnaire data from the US EPA subset were collected as part of a cross sectional study of chronic infections conducted in 2013 at the US EPA Human Studies Facility in Chapel Hill, NC. For the NIEHS subset, recruitment and data collection were conducted at the NIEHS facility (Research Triangle Park, NC) in 2013. Aliquots of serum samples and questionnaire data from the NIEHS subset were shared with the US EPA. Collected serum samples were archived at $-80^{\circ} \mathrm{C}$ until they were assayed for this study between late 2016 and early 2017.

The present study involved laboratory analysis of both subsets of samples for anti-HCMV IgG and analysis of biomarkers of vascular injury in the NIEHS subset. Samples from the US EPA subset have previously been analyzed using the same methods as the present study for multiple biomarkers of health including biomarkers of inflammation and endothelial function/vascular injury under research projects on the health effects of urban green spaces (Egorov et al., 2017, 2018).

\section{Questionnaire Data and Health Examinations}

Participants reported their demographic information (age, gender, race, ethnicity) and smoking history. However, only the US EPA survey included information on education level. None of the surveys collected data on income or assets. There were differences in smoking history questions between NIEHS and US EPA surveys: "current smoker" was defined as a participant who had smoked in the past $24 \mathrm{~h}$ (NIEHS) or who reported being a current smoker (US EPA). For the purpose of the analysis in this study, smoking data from both surveys were combined into a single binary variable (smoker vs. non-smoker). Height and weight data were measured by clinical staff at the US EPA or NIEHS facilities. Body Mass Index (BMI) was calculated using the measured height and weight of participants and categorized using the World Health Organization (WHO) standard definitions for BMI categories: underweight, normal, overweight and obese (WHO, 2000).

\section{Laboratory Analysis}

Serum samples were tested for IgG antibody response to HCMV using a commercially available ELISA kit (cat\# ab108724, Abcam, Cambridge, MA) following manufacturer's instructions. Serum samples were diluted 1:100 in the manufacturer provided sample diluent prior to being assayed. Twenty percent of samples were assayed in replicate on the same or different plates. The acceptable level of the coefficient of variation was set below 20\%; results not conforming with this requirement were excluded from statistical analysis. Blank corrected optical density (OD) values were divided by the plate specific cutoff value. Seropositive samples were defined as those with HCMV ratio to plate specific cutoff value $>1.1$ according to the manufacturer's instruction. Samples with inconclusive results (HCMV ratio to plate specific cutoff value $0.9-1.1$ ) were retested. For a single sample that remained inconclusive after several retests the result was dichotomized using the average HCMV ratio of 1.0 as a cutoff.

Serum CRP, SAA, sVCAM-1, and sICAM-1 levels were analyzed using a commercially available electrochemiluminescence Meso Scale Discovery (MSD) quadruplex Vascular Injury Panel 2 microplate assay (cat\# K15198D, MSD, Rockville, MD) following manufacturer's instructions. Serum samples were centrifuged at $2000 \times g$ for $3 \mathrm{~min}$ and then diluted 1:1,000 in the assay diluent prior to analysis. Twenty percent of samples were assayed in replicate on the same or different plates. The acceptable level of the coefficient of variation was set below 20\%; results not conforming with this requirement were excluded from statistical analysis. Responses were measured using an MSD QuickPlex SQ 120 instrument. Concentrations of the analytes were estimated from fourparameter logistic regression models fitted to serially diluted standards, as per manufacturer's instructions.

\section{Statistical Data Analysis}

$\mathrm{R}$ (version 3.5.1) and SAS (version 9.4) statistical analysis software packages were used to analyze the survey and assay data. Shapiro-Wilks test for normality and quantilequantile (Q-Q) plots were used to determine if logarithmic transformations were warranted. All four biomarkers of vascular injury (sICAM-1, sVCAM-1, CRP, and SAA), as well as BMI data were log-transformed to satisfy assumptions of normality. Race and ethnicity data were combined and dichotomized as non-Hispanic whites vs. all others. Race and ethnicity data were dichotomized this way because representation from other race categories in this study was not sufficient to make statistical comparisons. Missing values for covariates were imputed using the SAS procedure $m i$ for arbitrary missing data based on the multivariate discriminant function method.

Next, a multivariate predictive logistic regression model was developed for HCMV seropositivity. Covariates that were significant predictors $(p<0.05)$ of HCMV seropositivity in this model (age, BMI, gender, race/ethnicity, smoking status) were also incorporated in subsequent regression models to analyze associations between HCMV serostatus and vascular injury biomarkers.

The association between HCMV serostatus and levels of vascular injury biomarkers were investigated using linear regression models adjusted for covariates (SAS procedure genmod). Two alternative approaches were used. First, a binary variable for HCMV serostatus was used to assess the effects of seropositivity (Model 1). Second, anti-HCMV IgG antibody data were categorized as seronegative (reference category); or as 1st, 2nd, 3rd, or 4th quartiles of antibody responses in seropositive individuals 
in order to investigate whether there was an association between the intensity of anti-HCMV IgG responses and the levels of vascular injury biomarkers (Model 2). All results were expressed as percent changes in the median biomarker values.

Additional analysis under the second approach was conducted using the SAS procedure glm with the contrast statement in order to test for a linear trend in the effect of the intensity of anti-HCMV IgG antibody response on vascular injury biomarkers. This analysis was conducted using the ordinal variable for anti-HCMV IgG response as described above and assuming equal distances between all adjacent categories.

\section{RESULTS}

Serum samples from 694 of 703 study participants (99\%) were tested for anti-HCMV IgG response and all four biomarkers of vascular injury. Nine samples could not be analyzed due to low sample volumes. Seroprevalence of HCMV was $56 \%$ (Table 1). All samples retested on different plate lots produced consistent results. The age of participants ranged from 18 to 85 years. Almost two-thirds (62\%) of participants were women. A majority of participants (51\%) were non-Hispanic whites while $16 \%$ of participants declined to report their race. Almost a quarter (23\%) of participants reported a habit of smoking. Almost twothirds of participants (66\%) were overweight or obese (BMI

TABLE 1 | Descriptive statistics for the study population.

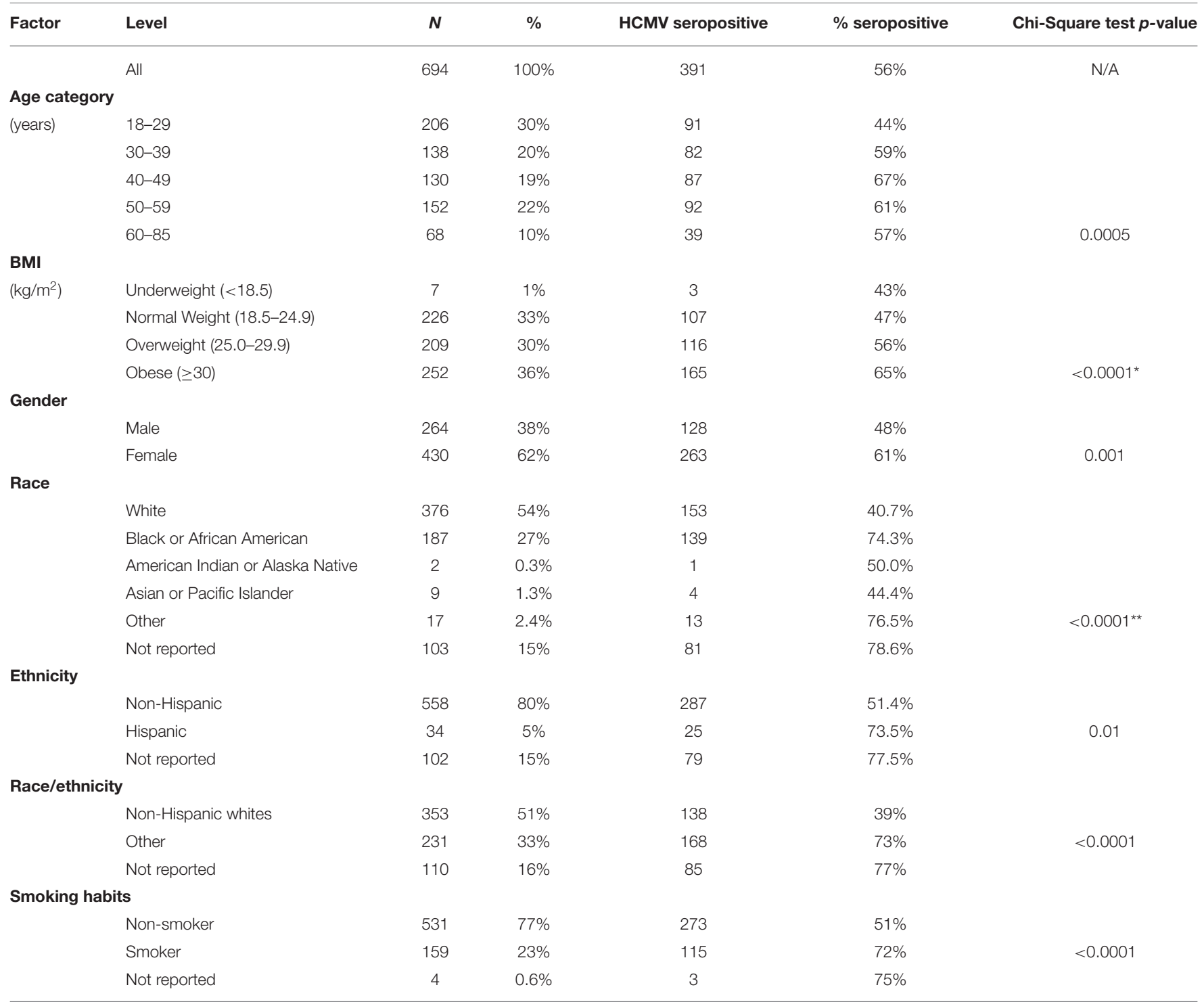

N/A, not applicable.

${ }^{*}$ Cochran-Armitage trend test.

${ }^{\star \star}$ Non-Hispanic White vs. all other races/ethnicities. 
$\geq 25.0 \mathrm{~kg} / \mathrm{m}^{2}$ ). Age, BMI, gender, smoking, and race/ethnicity status were significantly associated with HCMV seropositivity in exploratory univariate analysis (Table $\mathbf{1}$ ).

For regression analysis, missing race/ethnicity data $(n=110)$ and missing smoking data $(n=4)$ were imputed as described in the Methods section. Results of a multivariate predictive regression analysis for HCMV seropositivity demonstrated that increased age, female gender, racial/ethnic minority status, smoking, and greater BMI were significant predictors of HCMV seropositivity (Table 2). Specifically, a 10-year increase in age was associated with 1.25 (95\% CI 1.11-1.41) adjusted odds ratio (aOR) of seropositivity. Women had 2.06 (1.47-2.89) aOR of being seropositive compared to men. Non-white and/or Hispanic individuals had $2.90(2.03-4.13)$ aOR of being seropositive compared to non-Hispanic whites. Current smokers had 2.32 (1.54-3.51) aOR of seropositivity compared to non-smokers.

TABLE 2 | Effects of demographic predictors on HCMV seropositivity-adjusted odds ratios (OR) with a 95\% confidence interval from a multivariate logistic regression model.

\begin{tabular}{llc}
\hline Predictor & Level & Adjusted OR (95 \% Cl) \\
\hline Age (per 10-year increase) & NA & $1.25(1.11,1.41)$ \\
Gender & Males & Reference \\
& Females & $2.06(1.47,2.89)$ \\
Race/ethnicity & non-Hispanic whites & Reference \\
& All others & $2.90(2.03,4.13)$ \\
Smoking & Non-smoker & Reference \\
& Smoker & $2.32(1.54,3.51)$ \\
BMl (per 100\% increase) & NA & $1.72(1.02,2.88)$
\end{tabular}

For regression analysis, BMI data were $\log _{2}$-transformed; thus, the effect estimate is expressed as $1.72(1.02-2.88)$ aOR of seropositivity per doubling of BMI values.

HCMV seropositivity was associated with significantly increased levels of sICAM-1 and CRP after adjusting for covariates (Table 3). Median adjusted sICAM-1 and CRP levels in seropositive individuals were $9.2 \%(4.5-15 \%)$ and $20 \%(0.3-44 \%)$ higher than in seronegative controls, respectively.

In addition, greater intensity of anti-HCMV IgG antibody responses was significantly associated with increased levels sICAM-1 and sVCAM-1 (Table 3). Individuals in the top quartile of anti-HCMV IgG antibody responses had 16\% (8.0-25\%) and $7.7 \%(0.6-15 \%)$ higher median levels of sICAM-1 and sVCAM1 , respectively, compared to seronegative controls. Tests for linear trend using ordinal data on anti-HCMV IgG responses produced significant results for both sICAM-1 and sVCAM-1. Dose-response associations between the intensity of anti-HCMV IgG antibody response and biomarker level were not observed for CRP nor SAA.

\section{DISCUSSION}

We observed that HCMV seropositivity increased with greater age and BMI, and was more common among nonwhite and/or Hispanic individuals, women and smokers. Associations between HCMV seropositivity and increased age, race/ethnicity, and female gender are consistent with previously published results (Bate et al., 2010; Cannon et al., 2010; Simanek et al., 2011). Smoking, which is associated with lower socioeconomic status (Hiscock et al., 2012), has also been controlled for in previous studies of HCMV and CVD (Simanek et al., 2011).

TABLE 3 | Multiplicative effect estimates (95\% confidence intervals) of HCMV seropositivity or the intensity of anti-HCMV IgG antibody responses on vascular injury biomarkers. All models were adjusted for age, BMl, smoking status, gender, and race/ethnicity.

\begin{tabular}{|c|c|c|c|c|c|c|c|c|c|}
\hline \multirow[t]{2}{*}{ Model } & \multirow{2}{*}{$\begin{array}{l}\text { CMV serostatus, } \\
\text { median value } \\
\text { (range of values) }^{\star \star}\end{array}$} & \multicolumn{2}{|c|}{ SICAM-1 } & \multicolumn{2}{|c|}{ sVCAM-1 } & \multicolumn{2}{|c|}{ CRP } & \multicolumn{2}{|c|}{ SAA } \\
\hline & & $\begin{array}{c}\text { Effect estimate } \\
\text { (\% change) }\end{array}$ & $\begin{array}{l}p \text {-value for } \\
\text { trend }\end{array}$ & $\begin{array}{c}\text { Effect estimate } \\
\text { (\% change) }\end{array}$ & $\begin{array}{l}p \text {-value for } \\
\text { trend }\end{array}$ & $\begin{array}{c}\text { Effect estimate } \\
\text { (\% change) }\end{array}$ & $\begin{array}{l}p \text {-value for } \\
\text { trend }\end{array}$ & $\begin{array}{c}\text { Effect estimate } \\
\text { (\% change) }\end{array}$ & $\begin{array}{c}p \text {-value for } \\
\text { trend }\end{array}$ \\
\hline \multirow[t]{2}{*}{ 1. Seropositivity } & $\begin{array}{c}\text { Negative } 0.38 \\
(0.13-1.07)\end{array}$ & Reference & & Reference & & Reference & & Reference & \\
\hline & $\begin{array}{c}\text { Positive all } 3.86 \\
(1.10-10.97)\end{array}$ & $9.2(4.1,15)$ & & $2.2(-2.3,6.9)$ & & $20(0.3,44)$ & & $11(-7.4,32)$ & \\
\hline \multirow{5}{*}{$\begin{array}{l}\text { 2. Intensity of } \\
\text { anti-HCMV IgG } \\
\text { response }\end{array}$} & $\begin{array}{c}\text { Negative } 0.38 \\
(0.13-1.07)\end{array}$ & Reference & & Reference & & Reference & & Reference & \\
\hline & $\begin{array}{c}\text { Positive 1st quartile } \\
2.14(1.10-2.68)\end{array}$ & $6.9(-0.3,15)$ & & $-0.9(-7.1,5.8)$ & & $28(-1.5,67)$ & & $29(-0.4,66)$ & \\
\hline & $\begin{array}{l}\text { Posit. 2nd quartile } \\
3.26 \text { (2.69-3.86) }\end{array}$ & $8.7(1.5,17)$ & & $1.9(-4.5,8.6)$ & & $35(4.4,76)$ & & $-1.8(-24,27)$ & \\
\hline & $\begin{array}{c}\text { Posit. 3rd quartile } \\
4.40(3.87-4.86)\end{array}$ & $6.6(-0.6,14)$ & & $1.3(-5.1,8.2)$ & & $-3.1(-26 ; 26)$ & & $-2.6(-25,26)$ & \\
\hline & $\begin{array}{l}\text { Posit. 4th quartile } \\
5.63(4.90-10.97)\end{array}$ & $16(8.0,25)$ & 0.0008 & $7.7(0.6,15)$ & 0.04 & $23(-6.5,62)$ & 0.7 & $23(-5.8,61)$ & 0.7 \\
\hline
\end{tabular}

\footnotetext{
**Test values represent the ratio of optical densities of the sample and microplate-specific control.
} 
Regression models were adjusted for the above sociodemographic covariates, and two models (previously described) were used to explore associations between anti-HCMV IgG responses and levels of vascular injury and inflammation biomarkers. HCMV-seropositive individuals had significantly higher levels of sICAM-1 and CRP than seronegative controls. In addition, significant linear trends were observed between the intensity of anti-HCMV antibody responses and levels of sICAM-1 and sVCAM-1, but not levels of CRP. The observed association between HCMV serostatus and SAA was positive but not statistically significant, and there was no linear trend for this biomarker.

Results of this study corroborated previously observed associations between HCMV seropositivity and serum CRP levels (Fateh-Moghadam et al., 2003; Betjes et al., 2007; Simanek et al., 2011). To our knowledge, this was the first epidemiological study in the general population to show associations between antiHCMV IgG responses and vascular injury biomarkers sICAM1 and sVCAM-1. Previous research has only demonstrated the effects of HCMV on these cellular adhesion molecules in vitro, as well as in renal transplant recipients and individuals with symptomatic primary HCMV infection (Nordoy et al., 2000; Eriksson et al., 2001; Popović et al., 2012).

VCAM-1 and ICAM-1 play important roles in the vascular endothelium (Zakynthinos and Pappa, 2009). HCMV has been detected in endothelial cells (Crough and Khanna, 2009; Terrazzini et al., 2014). It has been proposed that the disruption of endothelial cells by HCMV triggers induction of proinflammatory adhesion molecules sICAM-1 and sVCAM-1, which potentially contributes to atherosclerosis and atherothrombosis (Popović et al., 2012). Our finding that elevated HCMV IgG response is associated with increased serum levels of sICAM-1 and sVCAM-1 provides further support for this hypothesis.

Previous epidemiological research demonstrated that exposure to common air pollutants was associated with increased levels of CRP, sICAM-1 and sVCAM-1 (Bind et al., 2012). Air pollution has been shown to cause adverse impacts on the cardiovascular system including endothelial dysfunction, atherosclerosis and thrombosis leading to increased risks of cardiovascular morbidity and mortality (An et al., 2018; Tibuakuu et al., 2018). Therefore, the associations between HCMV and vascular injury biomarkers observed in this study suggest that HCMV infection may increase susceptibility to detrimental health effects of air pollution. Our results suggest that in future investigations of the relationship between air pollution and CVD risk, it would be beneficial to control for HCMV serostatus due to the high prevalence of HCMV infection.

This cross-sectional study involved collecting information on common socio-demographic confounders (variables that are associated with both HCMV and vascular injury biomarkers) which have been controlled for in previous studies of HCMV infections. However, unaccounted confounders might have biased the observed associations between HCMV seropositivity and increased levels of vascular injury biomarkers. Although our regression analysis adjusted for age (a common predictor of chronic herpesvirus infections) and other demographic factors, we cannot completely rule out that the observed associations were partially confounded by other herpesviruses, such as Epstein-Barr virus (EBV).

Anti-HCMV IgM serum antibodies were not measured in the present study due to the low likelihood of detecting active primary HCMV infections in this cross-sectional sample of adult US residents. Previous research demonstrated that the incidence rate in the US population ages $12-49$ was $\sim 1.6$ infections per 100 susceptible person-years in 1988-1994 (Colugnati et al., 2007). Therefore, we are unable to conclude that all anti-HCMV IgG positive results were due to latent infection, even though it was the most likely stage of infection that the HCMV seropositive participants were experiencing.

The IgG test kits used in this study had high reported sensitivity (98\%) and specificity (97.5\%). Thus, false-positive responses and false-negative responses were unlikely to have a substantial impact on the observed associations of anti-HCMV IgG responses with vascular injury biomarkers.

The strength of our study lies in demonstrating positive associations between the intensity of anti-HCMV IgG antibody responses and vascular injury biomarkers sICAM-1 and sVCAM1. Arguably, socioeconomic factors, such as income and education, are less likely to confound these associations. However, it has been shown that the HCMV reactivation rate increases with age and is higher in women than in men (van Boven et al., 2017). While age and gender were controlled for in regression models in this study, there might be hypothetical unmeasured behavioral or life-style confounding factors that affected both the frequency of HCMV reactivation and the levels of vascular injury biomarkers. Further research which follows participants over time is warranted in order to confirm an association between HCMV reactivation and levels of vascular injury biomarkers, and to elucidate the triggers of HCMVrelated inflammation.

\section{CONCLUSION}

We observed associations between HCMV serostatus, intensity of anti-HCMV IgG responses, and vascular injury biomarkers sVCAM-1, sICAM-1, and CRP, which are known predictors of adverse cardiovascular outcomes. These findings suggest that a HCMV infection may contribute to the development of atherosclerosis and CVD.

\section{DATA AVAILABILITY STATEMENT}

The datasets for this article are not publicly available because they contain personally identifiable information (PII) collected with institutional review board review and approval which included an informed consent process which indicated individuals would not be identified as a result of their participation. Requests to access these datasets should be directed to Timothy J. Wade, wade.tim@epa.gov. 


\section{ETHICS STATEMENT}

This study involving human participants were reviewed and approved by the University of North Carolina at Chapel Hill Institutional Review Board. The patients/participants provided their written informed consent to participate in this study.

\section{AUTHOR CONTRIBUTIONS}

JNS, AE, RC, SG, and TW designed the study. RC, EH, and ES performed sample processing and contributed to data collection. AE, RC, TW, EH, and ES organized the study. JNS, SG, and EK carried out the laboratory experiments. JNS and AE performed data analysis. JNS, AE, LN-F, JRS, and TW drafted the manuscript. All authors contributed edits and comments.

\section{REFERENCES}

An, Z., Jin, Y., Li, J., Li, W., and Wu, W. (2018). Impact of particulate air pollution on cardiovascular health. Curr. Allergy Asthma Rep. 18:15. doi: $10.1007 / \mathrm{s} 11882-018-0768-8$

Bate, S. L., Dollard, S. C., and Cannon, M. J. (2010). Cytomegalovirus seroprevalence in the United States: the national health and nutrition examination surveys, 1988-2004. Clin. Infect. Dis. 50, 1439-1447. doi: $10.1086 / 652438$

Baumann, R., Gube, M., Markert, A., Davatgarbenam, S., Kossack, V., Gerhards, B., et al. (2017). Systemic serum amyloid A as a biomarker for exposure to zinc and / or copper-containing metal fumes. J. Expo. Sci. Environ. Epidemiol. 28, 84-91. doi: 10.1038/jes.2016.86

Betjes, M. G. H., Litjens, N. H. R., and Zietse, R. (2007). Seropositivity for cytomegalovirus in patients with end-stage renal disease is strongly associated with atherosclerotic disease. Nephrol. Dial. Transplant. 22, 3298-3303. doi: 10.1093/ndt/gfm 348

Bind, M. A., Baccarelli, A., Zanobetti, A., Tarantini, L., Suh, H., Vokonas, P., et al. (2012). Air pollution and markers of coagulation, inflammation, and endothelial function: associations and epigene-environment interactions in an elderly cohort. Epidemiology 23, 332-340. doi: 10.1097/EDE.0b013e31824523f0

Blankenberg, S., Barbaux, S., and Tiret, L. (2003). Adhesion molecules and atherosclerosis. Atherosclerosis 170, 191-203. doi: 10.1016/S0021-9150(03)00097-2

Cannon, M. J., Schmid, D. S., and Hyde, T. B. (2010). Review of cytomegalovirus seroprevalence and demographic characteristics associated with infection. Rev. Med. Virol. 20, 202-213. doi: 10.1002/rmv.655

Carlson, A., Norwitz, E. R., and Stiller, R. J. (2010). Cytomegalovirus infection in pregnancy: should all women be screened? Rev. Obstet. Gynecol. 3, 172-179.

Collins-Mcmillen, D., Buehler, J., Peppenelli, M., and Goodrum, F. (2018). Molecular determinants and the regulation of human cytomegalovirus latency and reactivation. Viruses 10:444. doi: 10.3390/v10080444

Colugnati, F. A., Staras, S. A., Dollard, S. C., and Cannon, M. J. (2007). Incidence of cytomegalovirus infection among the general population and pregnant women in the United States. BMC Infect. Dis. 7:71. doi: 10.1186/1471-2334-7-71

Crough, T., and Khanna, R. (2009). Immunobiology of human cytomegalovirus : from bench to bedside. Clin. Microbiol. Rev. 22, 76-98. doi: 10.1128/CMR.00034-08

Dollard, S. C., Grosse, S. D., and Ross, D. S. (2007). New estimates of the prevalence of neurological and sensory sequelae and mortality associated with congenital cytomegalovirus infection. Rev. Med. Virol. 17, 355-363. doi: 10.1002/ rmv. 544

Egorov, A. I., Converse, R., Griffin, S. M., Styles, J., Klein, E., Sams, E., et al. (2018). Environmental risk factors for Toxoplasma gondii infections and the impact of latent infections on allostatic load in residents of Central North Carolina. BMC Infect. Dis. 18:421. doi: 10.1186/s12879-018-3343-y

\section{FUNDING}

JNS was funded by the US EPA-UNC-CH Cooperative Training Agreement CR-83591401-0 and Cooperative Training Agreement CR-8357850-1, with the Department of Environmental Sciences and Engineering, the University of North Carolina at Chapel Hill.

\section{ACKNOWLEDGMENTS}

The authors are grateful to Dr. Stavros Garantziotis and his colleagues at the National Institute of Environmental Health Sciences (Research Triangle Park, North Carolina, USA) for providing aliquots of serum samples and questionnaire data on participants of the NIEHS study 10-E-0063.

Egorov, A. I., Griffin, S. M., Converse, R. R., Styles, J. N., Sams, E. A., Wilson, A., et al. (2017). Vegetated land cover near residence is associated with reduced allostatic load and improved biomarkers of neuroendocrine, metabolic and immune functions. Environ. Res. 158, 508-521. doi: 10.1016/j.envres.2017.07.009

Eriksson, B. M., Sjolin, J., Claesson, K., Wirgart, B. Z., Grillner, L., Totterman, T. H., et al. (2001). Circulating soluble vascular cell adhesion molecule1 and intercellular adhesion molecule-1 in immunocompetent and renal transplant patients: correlation with cytomegalovirus disease and renal function. Scand. J. Infect. Dis. 33, 350-354. doi: 10.1080/003655401750 173968

Fateh-Moghadam, S., Bocksch, W., Wessely, R., Jäger, G., Hetzer, R., and Gawaz, M., et al. (2003). Cytomegalovirus infection status predicts progression of heart-transplant vasculopathy. Transplantation 76, 1470-1474. doi: 10.1097/01.TP.0000090163.48433.48

Galkina, E., and Ley, K. (2007). Vascular adhesion molecules in atherosclerosis. Arterioscler. Thromb. Vasc. Biol. 27, 2292-2301. doi: 10.1161/ATVBAHA.107.149179

Haider, A. W., Wilson, P. W., Larson, M. G., Evans, J. C., Michelson, E. L., Wolf, P. A., et al. (2002). The association of seropositivity to Helicobacter pylori, Chlamydia pneumoniae, and cytomegalovirus with risk of cardiovascular disease: a prospective study. J. Am. Coll. Cardiol. 40, 1408-1413. doi: 10.1016/S0735-1097(02)02272-6

Hiscock, R., Bauld, L., Amos, A., Fidler, J. A., and Munafò, M. (2012). Socioeconomic status and smoking: a review. Ann. N. Y. Acad. Sci. 1248, 107-123. doi: 10.1111/j.1749-6632.2011.06202.x

Kaptoge, S., Angelantonio, E. D., and Pennells, L. (2012). C-reactive protein, fibrinogen, and cardiovascular disease prediction. N. Engl. J. Med. 1310-1320. doi: 10.1056/NEJMoa1107477

Lancini, D., Faddy, H. M., Flower, R., and Hogan, C. (2014). Cytomegalovirus disease in immunocompetent adults. Med. J. Aust. 201, 578-580. doi: $10.5694 / \mathrm{mja} 14.00183$

Lee, S., Brook, E., Affandi, J., Howson, P., Tanudjaja, S. A., Dhaliwal, S., et al. (2019). A. high burden of cytomegalovirus marks poor vascular health in transplant recipients more clearly than in the general population. Clin. Transl. Immunol. 8:e1043. doi: 10.1002/cti2.1043

Martin, J. A., Hamilton, B. E., Sutton, P. D., Ventura, S. J., Matthews, T., Kirmeyer, S., et al. (2010). Births: final data for 2007. Nat. Vital Stat. Rep. 58, 1-85.

Mehta, S. K., Stowe, R. P., Feiveson, A. H., Tyring, S. K., and Pierson, D. L. (2000). Reactivation and shedding of cytomegalovirus in astronauts during spaceflight. J. Infect. Dis. 182, 1761-1764. doi: 10.1086/317624

Nordoy, I., Muller, F., Nordal, K. P., Rollag, H., Aukrust, P., Froland, S. S., et al. (2000). Chemokines and soluble adhesion molecules in renal transplant recipients with cytomegalovirus infection. Clin. Exp. Immunol. 120, 333-337. doi: 10.1046/j.1365-2249.2000. 01221.x 
Popović, M., Smiljanić, K., Dobutović, B., Syrovets, T., Simmet, T., Isenović, E. R., et al. (2012). Human cytomegalovirus infection and atherothrombosis. J. Thromb. Thrombolysis. 33, 160-172. doi: 10.1007/s11239-011-0662-x

Prince, H. E., and Lapé-Nixon, M. (2014). Role of cytomegalovirus (CMV) IgG avidity testing in diagnosing primary CMV infection during pregnancy. Clin. Vaccine Immunol. 21, 1377-1384. doi: 10.1128/CVI.00487-14

Ross, S. A., and Boppana, S. B. (2004). Congenital cytomegalovirus infection: outcome and diagnosis. Semin. Pediatr. Infect. Dis. 16, 44-49. doi: 10.1053/j.spid.2004.09.011

Schleiss, M. R. (2008). Cytomegalovirus vaccine development. Curr. Top. Microbiol. Immunol. 325, 361-382. doi: 10.1007/978-3-540-77349-8_20

Simanek, A. M., Cheng, C., Yolken, R., Uddin, M., Galea, S., Aiello, A. E., et al. (2014). Herpesviruses, inflammatory markers and incident depression in a longitudinal study of Detroit residents. Psychoneuroendocrinology 50, 139-148. doi: 10.1016/j.psyneuen.2014.08.002

Simanek, A. M., Dowd, J. B., Pawelec, G., Melzer, D., Dutta, A., Aiello, A. E., et al. (2011). Seropositivity to cytomegalovirus, inflammation, all-cause and cardiovascular disease-related mortality in the United States. PLoS ONE 6:e16103. doi: 10.1371/journal.pone.0016103

Terrazzini, N., Bajwa, M., Thomas, D., Smith, H., and Kern, F. (2014). Gender differences and age-specific associations between body mass index and other cardiovascular risk factors in CMV infected and uninfected people. Immunol. Lett. 162, 316-322. doi: 10.1016/j.imlet.2014.09.011

Tibuakuu, M., Michos, E. D., Navas-Acien, A., and Jones, M. R. (2018). Air pollution and cardiovascular disease: a focus on vulnerable populations Worldwide. Curr. Epidemiol. Rep. 5, 370-378. doi: 10.1007/s40471-018-0166-8 van Boven, M., van de Kassteele, J., Korndewal, M. J., van Dorp, C. H., Kretzschmar, M., van Der Klis, F., et al. (2017). Infectious reactivation of cytomegalovirus explaining age- and sex-specific patterns of seroprevalence. PLoS Comput. Biol. 13:e1005719. doi: 10.1371/journal.pcbi. 1005719

WHO (2000). Obesity : Preventing and Managing the Global Epidemic. WHO Technical Report Series. Geneva: World Health Organization.

Yaiw, K. C., Ovchinnikova, O., Taher, C., Mohammad, A. A., Davoudi, B., Shlyakhto, E., et al. (2013). High prevalence of human cytomegalovirus in carotid atherosclerotic plaques obtained from Russian patients undergoing carotid endarterectomy. Herpesviridae 4:3. doi: 10.1186/2042-4280-4-3

Zakynthinos, E., and Pappa, N. (2009). Inflammatory biomarkers in coronary artery disease. J. Cardiol. 53, 317-333. doi: 10.1016/j.jjcc.2008.12.007

Disclaimer: The views expressed in this article are those of the authors and do not necessarily represent the views or policies of US EPA. Mention of trade names, products, or services does not convey, and should not be interpreted as conveying official US EPA approval, endorsement or recommendation.

Conflict of Interest: The authors declare that the research was conducted in the absence of any commercial or financial relationships that could be construed as a potential conflict of interest.

Copyright $\odot 2020$ Styles, Converse, Griffin, Wade, Klein, Nylander-French, Stewart, Sams, Hudgens and Egorov. This is an open-access article distributed under the terms of the Creative Commons Attribution License (CC BY). The use, distribution or reproduction in other forums is permitted, provided the original author $(s)$ and the copyright owner(s) are credited and that the original publication in this journal is cited, in accordance with accepted academic practice. No use, distribution or reproduction is permitted which does not comply with these terms. 